\title{
Usefulness of the updated logistic clinical SYNTAX score after percutaneous coronary intervention in patients with prior coronary artery bypass graft surgery: Insights from the GLOBAL LEADERS trial
}

\author{
Hironori Hara MD $\quad$ | Norihiro Kogame MD ${ }^{1} \quad$ Kuniaki Takahashi MD ${ }^{1} \quad$ | \\ Rodrigo Modolo MD ${ }^{1}$ ～Ply Chichareon MD ${ }^{1,2}$ | Mariusz Tomaniak MD ${ }^{3,4}$ (c) |

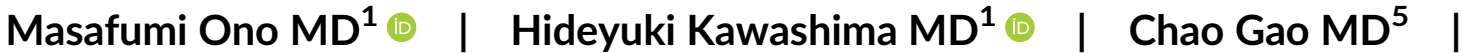 \\ Rutao Wang $M^{5} \quad$ ～Veselin D. Valkov $M^{6}$ ｜ Jürgen vom Dahl MD | \\ Clemens Steinwender MD $^{8}$ ｜ Tobias Geisler MD ${ }^{9}$ | \\ Pedro Alves Lemos Neto MD, PhD ${ }^{10,11}$ ｜Ｃarlos Macaya Miguel MD ${ }^{12}$ |
}

Scot Garg MD, PhD ${ }^{13}$ ｜ Peter Jüni MD ${ }^{14}$ | Christian Hamm MD ${ }^{15}$ |

Philippe Gabriel Steg MD ${ }^{16}$ | Marco Valgimigli MD, $\mathrm{PhD}^{17}$ [ । |

Pascal Vranckx MD, PhD ${ }^{18}$ | Stephan Windecker MD $^{17}$ | Vasim Farooq MD, PhD ${ }^{19}$ |

Yoshinobu Onuma MD, PhD ${ }^{20}$ | Patrick W. Serruys MD, PhD 20,21 | for the GLOBAL

LEADERS Trial Investigators

\footnotetext{
${ }^{1}$ Department of Cardiology, Academic Medical Center, University of Amsterdam, Amsterdam, The Netherlands

${ }^{2}$ Cardiology unit, Department of Internal Medicine, Faculty of Medicine, Prince of Songkla University, Songkhla, Thailand

${ }^{3}$ Department of Cardiology, Erasmus Medical Center, Erasmus University, Rotterdam, The Netherlands

${ }^{4}$ First Department of Cardiology, Medical University of Warsaw, Warsaw, Poland

${ }^{5}$ Department of Cardiology, Radboud University, Nijmegen, The Netherlands

${ }^{6}$ Department of Cardiology, St. Marina UMHAT, Varna, Bulgaria

${ }^{7}$ Klinik f. Kardiologie/Intern, Intensivmedizin, Kliniken Maria Hilf GmbH, Akademisches Lehrkrankenhaus der RWTH Aachen, Mönchengladbach, Germany

${ }^{8}$ Department of Cardiology, Kepler University Hospital Linz, Medical Faculty, Johannes Kepler University, Linz, Austria

${ }^{9}$ Department of Cardiology and Angiology, University Hospital Tübingen, Tübingen, Germany

${ }^{10}$ Heart Institute (InCor), University of Sao Paulo Medical School, São Paulo, Brazil

${ }^{11}$ Interventional Cardiology, Hospital Israelita Albert Einstein, São Paulo, Brazil

${ }^{12}$ Department of Cardiology, Instituto Cardiovascular, Hospital Clínico San Carlos, Universidad Complutense, Madrid, Spain

${ }^{13}$ Department of Cardiology, Royal Blackburn Hospital, Blackburn, United Kingdom

${ }^{14}$ Applied Health Research Centre (AHRC), Li Ka Shing Knowledge Institute of St. Michael's Hospital, Department of Medicine and Institute of Health Policy,

Management and Evaluation, University of Toronto, Toronto, Ontario, Canada

${ }^{15}$ Kerckhoff Heart Center, Campus University of Giessen, Bad Nauheim, Germany

${ }^{16}$ FACT (French Alliance for Cardiovascular Clinical Trials), DHU FIRE, INSERM Unité 1148, Université de Paris, and Hôpital Bichat, Assistance-Publique-Hôpitaux de Paris, Paris, France, and NHLI, Imperial College, Royal Brompton Hospital, London, United Kingdom

${ }^{17}$ Department of Cardiology, Bern University Hospital, Bern, Switzerland

${ }^{18}$ Department of Cardiology and Intensive Care, Jessa Ziekenhuis, Hasselt, Belgium; Faculty of Medicine and Life Sciences University of Hasselt, Hasselt, Belgium

${ }^{19}$ Manchester Heart Centre, Manchester Royal Infirmary, Central Manchester University Hospitals, Manchester, United Kingdom

${ }^{20}$ Department of cardiology, National University of Ireland Galway (NUIG), Galway, Ireland

${ }^{21} \mathrm{NHLI}$, Imperial College London, London, United Kingdom
} 


\section{Correspondence}

Patrick W. Serruys, MD, PhD, Established Professor of Interventional Medicine and Innovation, National University of Ireland Galway (NUIG), University Road, Galway H91 TK33, Ireland.

Email: patrick.w.j.c.serruys@gmail.com

\section{Funding information}

AstraZeneca; Biosensors International Group; Medicines Company

\begin{abstract}
Objectives: We aimed to investigate the prognostic utility of the anatomical CABG SYNTAX and logistic clinical SYNTAX scores for mortality after percutaneous coronary intervention $(\mathrm{PCl})$ in patients with prior coronary artery bypass grafts (CABG).
\end{abstract}

Background: The anatomical SYNTAX score evaluated the anatomical complexity of coronary artery disease and helped predict the prognosis of patients undergoing $\mathrm{PCl}$. The anatomical CABG SYNTAX score was derived from the anatomical SYNTAX score in patients with prior CABG, whilst the logistic clinical SYNTAX score was developed by incorporating clinical factors into the anatomical SYNTAX score.

Methods: We calculated the anatomical CABG SYNTAX score and logistic clinical SYNTAX score in 205 patients in the GLOBAL LEADERS trial. The predictive abilities of these scores for 2-year all-cause mortality were evaluated.

Results: Using the median scores as categorical thresholds between low and high score groups, the logistic clinical SYNTAX score was able to discriminate the risk of 2-year mortality, unlike the anatomical CABG SYNTAX score. The logistic clinical SYNTAX was significantly better at predicting 2-year mortality, compared to the anatomical CABG SYNTAX score, as evidenced by AUC values in receiver-operating characteristic curve analysis $(0.806$ vs. $0.582, p<.001)$ and integrated discrimination improvement $(0.121, p<.001)$.

Conclusions: The logistic clinical SYNTAX score was superior to the anatomical CABG SYNTAX score in predicting 2-year mortality.

\section{KEYWORDS}

coronary bypass grafts, drug eluting, percutaneous coronary intervention (PCI), risk stratification, stent

\section{1 | INTRODUCTION}

The anatomical SYNTAX (Synergy between Percutaneous Coronary Intervention with Taxus and Cardiac Surgery) score ${ }^{1}$ is recommended to evaluate the anatomical complexity of coronary artery disease and can also help predict medium-term prognosis in patients undergoing percutaneous coronary intervention $(\mathrm{PCl}) .^{2-4}$ The anatomical CABG SYNTAX score, which takes into account the extent of revascularization by bypass grafts, was proposed in 2012 for the patients who had undergone coronary artery bypass graft (CABG) surgery. ${ }^{5}$ However, the prognostic value of this specific score has not yet been further evaluated in patients with prior CABG undergoing $\mathrm{PCl}^{6}$

To enable individualized risk estimation for all-cause mortality after PCl, the clinical SYNTAX score and logistic clinical SYNTAX score was developed by combining the anatomical SYNTAX score and clinical factors, and the logistic clinical SYNTAX score has been recently updated and validated. ${ }^{7-10}$ The performance of the logistic clinical SYNTAX score has been assessed in patients with left main coronary artery disease and acute coronary syndromes, ${ }^{11,12}$ but not in patients with prior CABG undergoing $\mathrm{PCl}$.
We aimed to investigate and compare the prognostic performance of the anatomical CABG SYNTAX score, clinical SYNTAX score and logistic clinical SYNTAX score in predicting 2 years all-cause mortality after $\mathrm{PCl}$ in patients with prior CABG using the GLOBAL LEADERS trial database. ${ }^{13}$

\section{2 | METHODS}

\section{1 | Study design and participants}

The GLOBAL LEADERS trial (NCT01813435) was a prospective randomized, open-label trial, designed to compare 23-month ticagrelor monotherapy following one-month dual antiplatelet therapy and 12-month dual antiplatelet therapy followed by 12-month aspirin monotherapy after $\mathrm{PCl}$ in a total of 15,991 all-comers patients. ${ }^{13}$

The anatomical SYNTAX score analysis was prespecified in the protocol for the first 4,000 consecutive patients in the GLOBAL LEADERS trial. ${ }^{14}$ Among the first 4000 consecutive patients, 275 patients had a prior CABG. Of these 275 patients, one patient did 
not receive $\mathrm{PCl}$, and coronary arteries and bypass grafts were not fully assessed in 48 patients (e.g., native right coronary artery was not assessed since a bypass graft to right coronary artery was patent). Therefore, 226 had coronary angiograms for which the anatomical CABG SYNTAX score could be calculated by an independent core lab (ART, Academic Research Team, Rotterdam, The Netherlands), ${ }^{5}$ by analysts unaware of the patient's treatment assignment or clinical outcome.

The anatomical CABG SYNTAX score $^{5}$ derived from Leaman score $^{15}$ was calculated by determining the standard anatomical SYNTAX score in the "native" coronary vessels (native SYNTAX score) and deducting points based on the weighting of the diseased coronary artery segment that have a functioning bypass graft anastomosed distally. Therefore, the anatomical CABG SYNTAX score could reflect anatomical complexity and extent of revascularization, as well as the anatomical SYNTAX score after PCl. An example of the calculation is presented in Figure 1.

Of these 226 patients, at least one variable for the logistic clinical SYNTAX score calculation was missing in 21 patients, thus, all baseline characteristics for the updated logistic clinical SYNTAX score ${ }^{9}$ calculation including age, creatinine clearance $(\mathrm{CrCl})$, left ventricular ejection fraction (LVEF), body mass index (BMI), diabetes, peripheral vascular disease (PVD), and SYNTAX-like characteristics, were available in 205 patients. SYNTAX-like characteristic was defined as unprotected left main coronary artery disease and/or three vessel disease without patency of grafts.

The clinical SYNTAX score is one of the historical and developmental SYNTAX-derived scores and includes only three patient characteristics: age, $\mathrm{CrCl}$, and LVEF and was inspired by the ACEF score. ${ }^{16-19}$ This score was calculated using the following formula; (the anatomical CABG SYNTAX score) $\times$ (age/EF +1 point for every 10 reduction in $\mathrm{CrCl}$ below $60 \mathrm{~mL} / \mathrm{min}) .^{7}$ In this calculation, $\mathrm{a} \mathrm{CrCl}$ of between 50 and $59 \mathrm{~mL} / \mathrm{min}, 40$ and $49 \mathrm{~mL} / \mathrm{min}, 30$ and $39 \mathrm{~mL} / \mathrm{min}$, 20 and $29 \mathrm{~mL} / \mathrm{min}, 10$ and $19 \mathrm{~mL} / \mathrm{min}$, and 0 and $9 \mathrm{~mL} / \mathrm{min}$ would receive $1,2,3,4,5$, and 6 points, respectively.

The updated logistic clinical SYNTAX score in patients with prior CABG was calculated using the following formula; $0.0187 \times$ (the anatomical CABG SYNTAX score) $+0.1667 \times$ (SYNTAX-like characteristic) $+0.0425 \times$ (age) $+0.0174 \times(90-\mathrm{CrCl})+0.0522 \times(50-\mathrm{EF})$ $+0.0312 \times(\mathrm{BMI})+0.57 \times(\mathrm{PVD})+0.3463 \times$ (diabetes) -4.521 .

All patients provided informed, written consent. The study complied with the Declaration of Helsinki and Good Clinical Practices. (a)

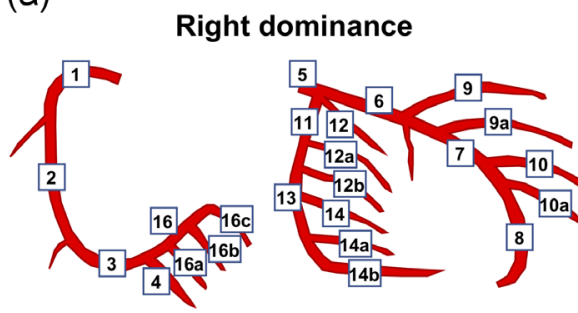

Left dominance
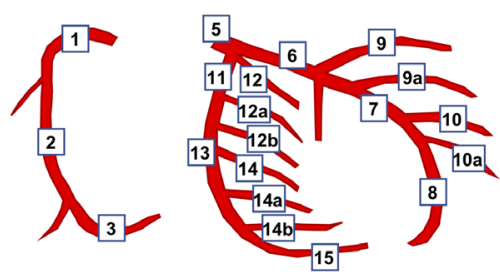

(b)

\begin{tabular}{cc} 
Lesion & $\begin{array}{c}\text { Segment-weighting } \\
\text { multiplication factors }\end{array}$ \\
\hline $\begin{array}{c}\text { Total occlusion } \\
\text { (Diameter stenosis: } 100 \% \text { ) }\end{array}$ & $\mathbf{5}$ \\
\hline $\begin{array}{c}\text { Significant stenosis } \\
\text { (Diameter stenosis: } 50-99 \%)\end{array}$ & $\mathbf{2}$ \\
\hline
\end{tabular}

(c)

\begin{tabular}{lcc} 
& Segment & Weighting \\
\hline RCA & $1,2,3,4$ & 1 \\
& $16,16 a, 16 b, 16 c$ & 0.5 \\
\hline LMCA & 5 & 5 \\
\hline LAD & 6 & 3.5 \\
& 7 & 2.5 \\
& $8,9 a, 9 b$ & 1 \\
& $10,10 a$ & 0.5 \\
\hline LCX & 11 & 1.5 \\
& $12,12 a, 12 b$ & 1 \\
& $13,14,14 a, 14 b$ & 0.5 \\
\hline
\end{tabular}

\begin{tabular}{lcc} 
& Segment & Weighting \\
\hline RCA & $1,2,3$ & 0 \\
\hline LMCA & 5 & 6 \\
LAD & 6 & 3.5 \\
& 7 & 2.5 \\
& $8,9 \mathrm{a}, 9 \mathrm{~b}$ & 1 \\
& $10,10 \mathrm{a}$ & 0.5 \\
\hline LCX & 11 & 2.5 \\
& $12,12 \mathrm{a}, 12 \mathrm{~b}$ & 1 \\
& 13 & 1.5 \\
& $14,14 \mathrm{a}, 14 \mathrm{~b}, 15$ & 1 \\
\hline
\end{tabular}

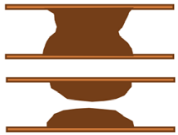

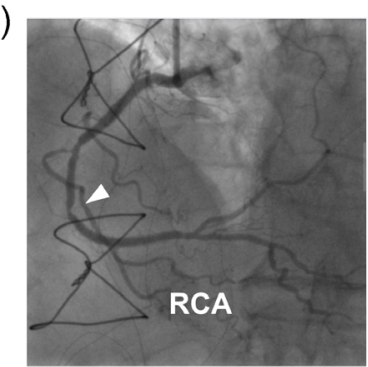

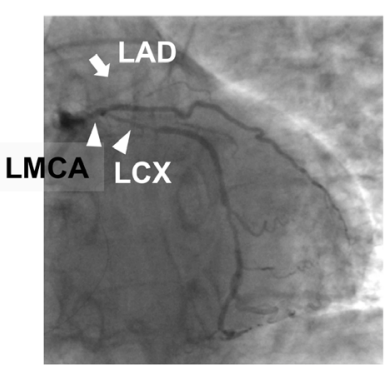

Native SYNTAX score: 41.5
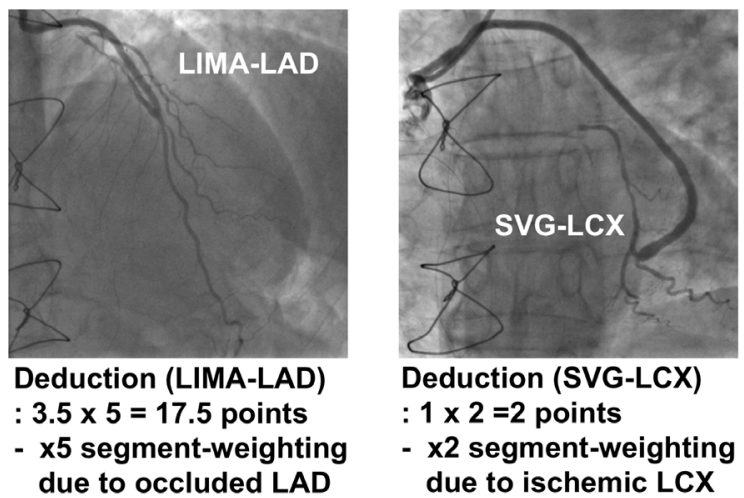

CABG SYNTAX score: 22

FIGURE 1 An example of the calculation of the anatomical CABG SYNTAX score. ( $a$ and $b$ ) Coronary segment-weighting derived from Leaman score ${ }^{5,15}$ (a), and segment-weighting multiplication factors depending on severity of the lesion ${ }^{15}$ (b). These were used to calculate points for deduction from the native SYNTAX score. (c) The native SYNTAX score was 41.5 due to left main and three-vessel disease (upper images). A patent left internal mammary artery (LIMA) to left anterior descending artery (LAD) with no intervening obstructive disease (lower left image) led to the deduction of $3.5 \times 5$ points ( $\times 5$ segment-weighting due to occluded LAD) from the native SYNTAX score. A patent saphenous vein graft (SVG) to left circumflex (LCX) with no intervening obstructive coronary disease (lower right image) led to $1 \times 2$ points ( $\times 2$ segment-weighting due to ischemic LCX) deduction. Therefore, the CABG SYNTAX score was 41.5-17.5-2 = 22 points. RCA, right coronary artery; LMCA, left main coronary artery; an arrow indicates occlusion; arrow heads indicate stenosis [Color figure can be viewed at wileyonlinelibrary.com] 


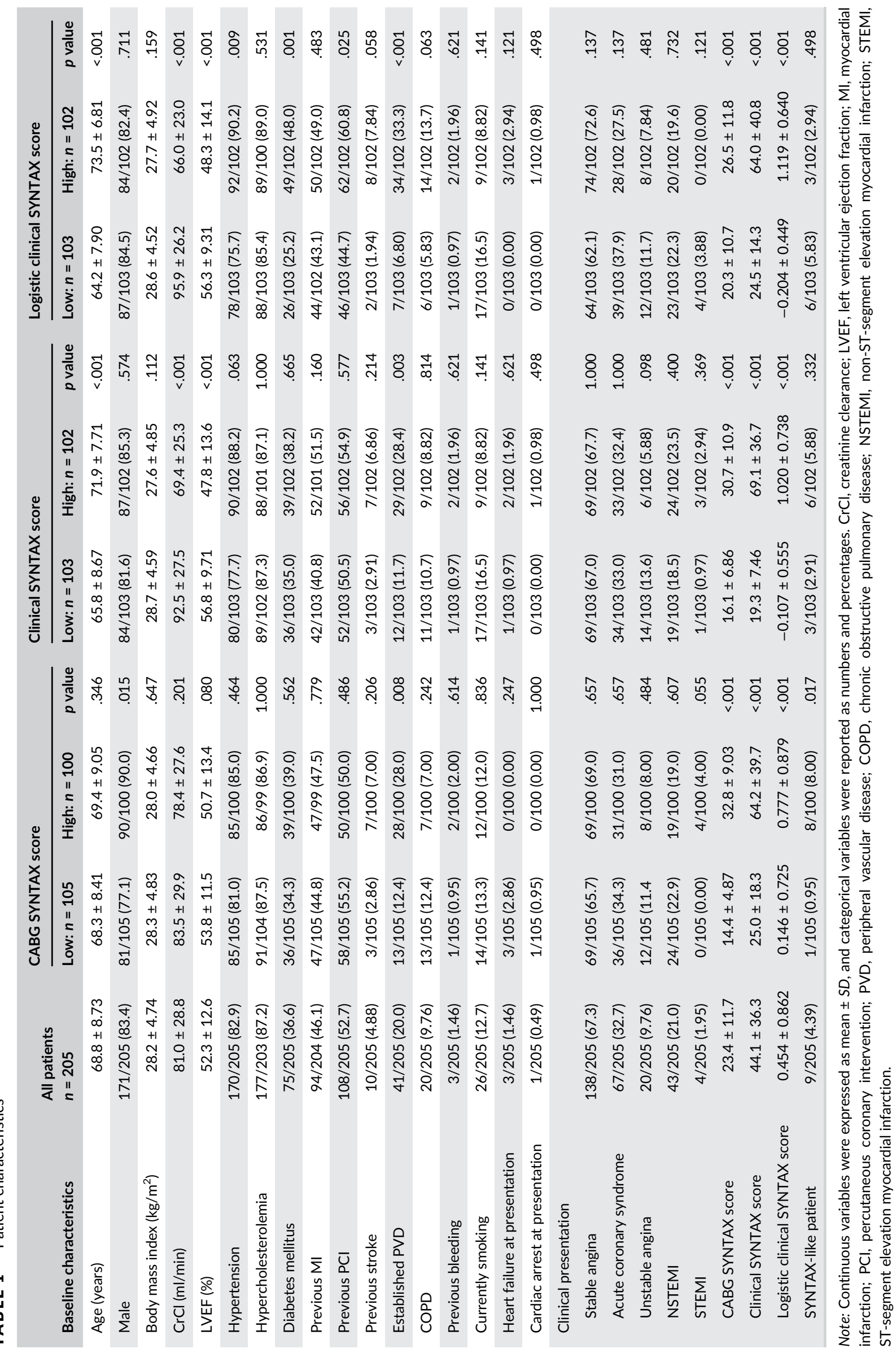



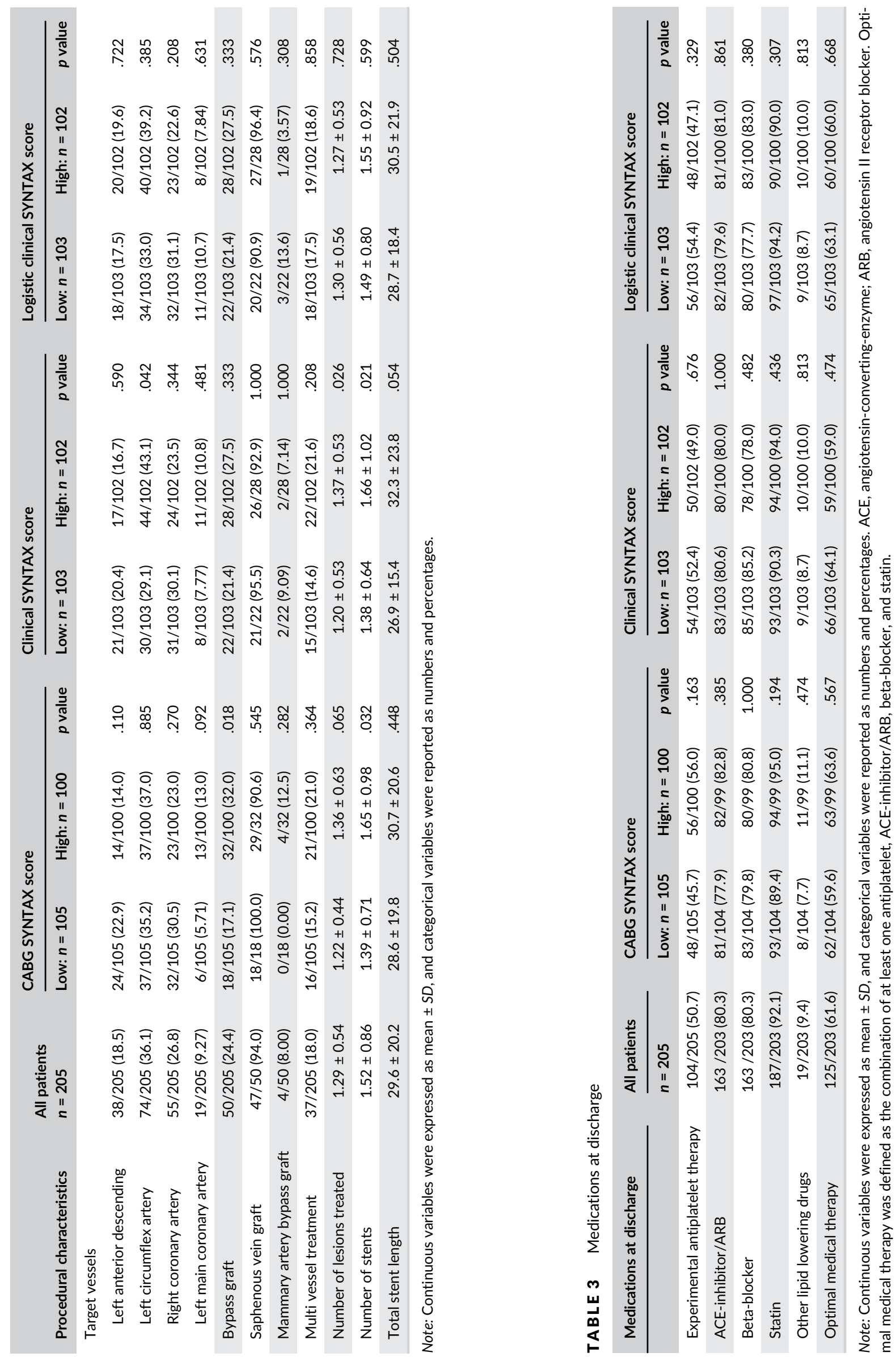
(a)

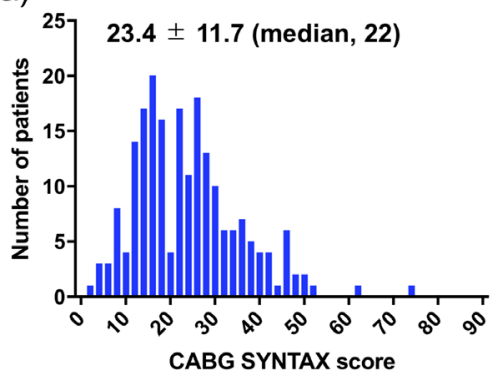

(b)

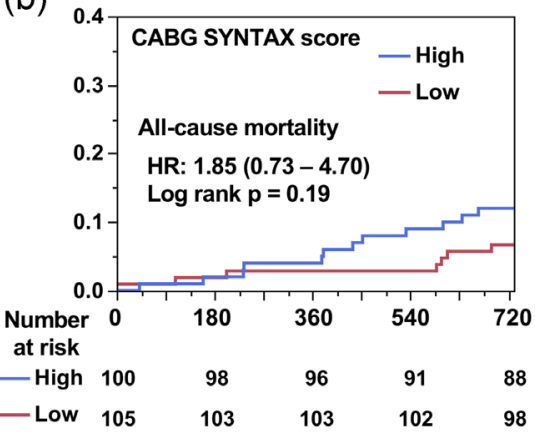

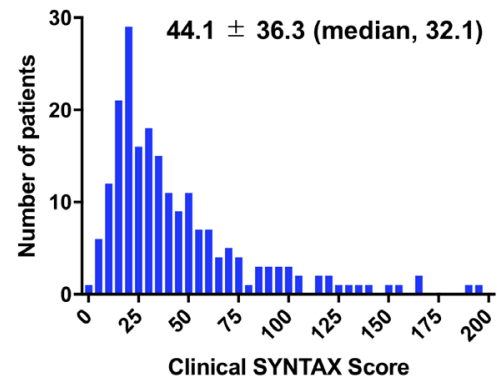

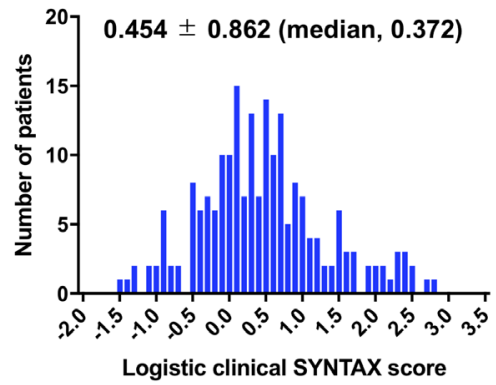

FIGURE 2 (a) Distribution of the anatomical CABG SYNTAX scores, clinical SYNTAX scores and logistic clinical SYNTAX scores. Scores are shown as mean $\pm S D$ (median). (b) Cumulative incidence of all-cause mortality at 2 years [Color figure can be viewed at wileyonlinelibrary.com]

(b)

(a)

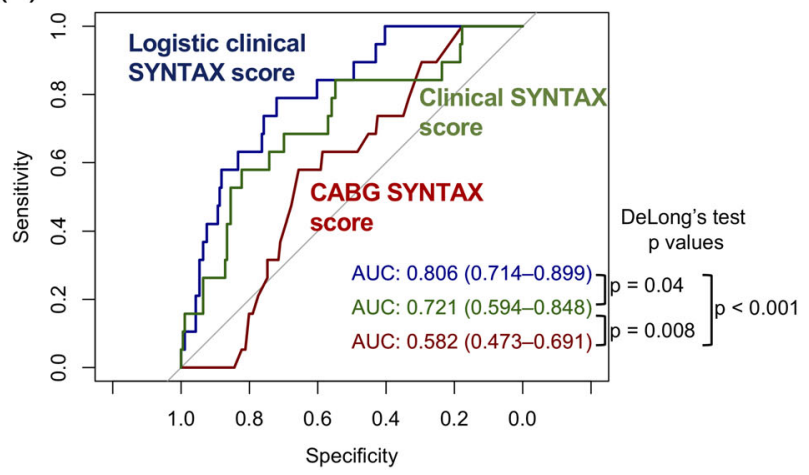

\section{(b)}

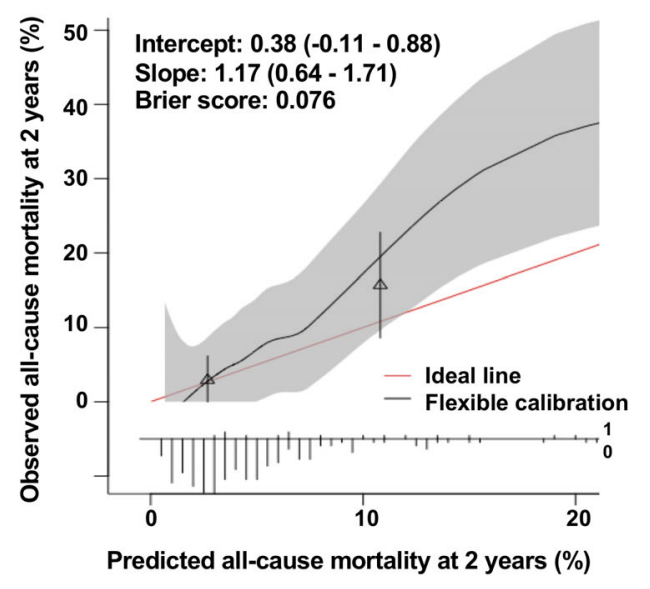

FI GURE 3 (a) Receiver-operating characteristic curves for the anatomical CABG SYNTAX score, clinical SYNTAX score and logistic clinical SYNTAX score predicting 2-year all-cause mortality. $p$ values were obtained using DeLong's test. (b) Calibration plot for the updated logistic clinical SYNTAX score for 2-year all-cause mortality. Triangles represent two groups of patients with mean predicted probability and mean observed all-cause mortality rate with $95 \%$ confidence interval. The distribution of patients is indicated with spike at the bottom of the graph, stratified by outcomes (deaths above the $x$-axis and survivors below the $x$-axis) [Color figure can be viewed at wileyonlinelibrary.com]

\subsection{Endpoint}

This study is a non-prespecified post-hoc analysis of the GLOBAL LEADERS trial. The primary endpoint of this study was 2-year allcause mortality. The causes of death were classified to cardiovascular death, noncardiovascular death and undetermined according to the Academic Research Consortium (ARC)-2 definition. ${ }^{20}$ As defined in the ARC-2 definition, undetermined death was classified as cardiovascular death for end point determination. Patient-oriented composite endpoint (POCE) of all-cause mortality, any stroke, any myocardial infarction or any revascularization, net adverse clinical events (NACE) of POCE or Bleeding Academic Research Consortium (BARC) grade 3 or 5 bleeding, and their components were also assessed with the scores that have been specifically designed to predict all-cause 
mortality. ${ }^{9,10}$ The survival status of the patients lost to follow up was obtained through public civil registry and more than $99.95 \%$ of the vital status at 2 years were available in the GLOBAL LEADERS trial. ${ }^{13}$ The vital status at 2 years were available in all 205 patients.

\subsection{Statistical analysis}

Continuous variables were expressed as mean $\pm S D$, and were compared using Student's $t$ test or Mann-Whitney $U$ test. Categorical variables were reported as numbers and percentages, and were compared using chi square or Fisher's exact test as appropriate. The cumulative event rates at 2 years were estimated by Kaplan-Meier method and comparisons of outcomes were performed with log-rank test. The all-cause mortality risk reclassification was assessed using the net reclassification index (NRI). The predictive capability of the anatomical CABG SYNTAX score, clinical STNTAX score and logistic clinical SYNTAX score for the 2-year outcomes was assessed using receiver-operating characteristic (ROC) curve analysis with area under the curve (AUC). DeLong's test was used to analyze the differences between AUC values of the anatomical CABG SYNTAX score, clinical SYNTAX score and logistic clinical SYNTAX score and the corresponding $p$ values. The predictive value was also assessed by integrated discrimination improvement (IDI). Agreement between

TABLE 4 Causes of death

\begin{tabular}{lllr} 
& & \multicolumn{3}{l}{$\begin{array}{l}\text { Logistic clinical } \\
\text { SYNTAX score }\end{array}$} \\
\cline { 3 - 4 } Causes of death & $\boldsymbol{n}=205$ & Low: $\boldsymbol{n}=103$ & High: $\boldsymbol{n}=102$ \\
\hline Cardiovascular death & $11 / 205(5.37)$ & $0 / 103(0.00)$ & $11 / 102(10.8)$ \\
$\begin{array}{l}\text { Noncardiovascular } \\
\text { death }\end{array}$ & $7 / 205(3.37)$ & $2 / 103(1.94)$ & $5 / 102(4.90)$ \\
\hline Undetermined & $1 / 205(0.48)$ & $1 / 103(0.97)$ & $0 / 102(0.00)$ \\
\hline
\end{tabular}

Note: Variables were reported as numbers and percentages.

TAB LE 5 Predictive ability of the logistic clinical SYNTAX score for outcomes at 2 years

\begin{tabular}{ll}
\hline Outcomes at 2 years & AUCs \\
\hline All-cause death & $0.806(0.714-0.899)$ \\
\hline Cardiovascular death & $0.825(0.717-0.934)$ \\
\hline Stroke & $0.668(0.354-0.982)$ \\
\hline Myocardial infarction & $0.674(0.487-0.861)$ \\
\hline Revascularization & $0.537(0.430-0.645)$ \\
\hline BARC 3 or 5 & $0.574(0.369-0.779)$ \\
\hline POCE & $0.590(0.496-0.685)$ \\
\hline NACE & $0.592(0.500-0.685)$ \\
\hline
\end{tabular}

Abbreviations: AUC, area under the curve; BARC, bleeding academic research consortium; POCE, patient-oriented composite endpoint; NACE, net adverse clinical events. observed and predicted all-cause mortality was assessed by calibration plot. Two groups based on the updated logistic clinical SYNTAX score were depicted in the calibration plot augmented by a locally weighted scatterplot smoothing. ${ }^{21}$ Calibration-in-the-large (model intercept) and calibration slope were evaluated by fitting the calculated linear predictor in all patients with all-cause mortality as the outcome in the logistic regression model. Intercept of 0 and slope of 1 indicate perfect prediction. Negative and positive intercepts indicate overestimation and underestimation, respectively. Brier score was reported as an overall measure of performance, which ranges from 0 (perfect model) to 0.25 (non-informative model). ${ }^{21}$

A two-sided $p$ value $<.05$ was considered statistically significant. Analyses were performed using JMP Pro14 (SAS Institute Inc., Cary, NC) and R version 3.6.0 (R Foundation for Statistical Computing, Vienna, Austria).

\section{$3 \mid$ RESULTS}

Patient characteristics, procedure characteristics, and medications at discharge are shown in Tables 1-3, respectively. Distributions of the anatomical CABG SYNTAX Scores, clinical STNTAX scores and logistic clinical SYNTAX scores are shown in Figure 2a. Patients were divided into two groups based on the median of the scores, as previously reported $^{5}$ (anatomical CABG SYNTAX score; $\leqq 22$ [low group, $n=105$ ], $>22$ [high group, $n=100$ ], clinical SYNTAX score; $\leqq 32.13$ [low group, $n=103$ ], $>32.13$ [high group, $n=102$ ] and logistic clinical SYNTAX score; $\leqq 0.372$ [low group, $n=103$ ], $>0.372$ [high group, $n=102$ ]). The rate of all-cause mortality at 2 years in the high anatomical CABG SYNTAX score group tended to be numerically higher than in the low anatomical CABG SYNTAX score group, although they were not significantly different (low $=6.7 \%$ vs. high $=12.0 \%, p=.19$, Figure $2 b$ ). On the other hand, there were significant differences in all-cause mortality at 2-year between patients in the high versus low clinical SYNTAX score group (low clinical SYNTAX score, 2.9\%; high clinical SYNTAX score, $15.7 \% ; p=.0017$ ) and those in the high versus low logistic clinical SYNTAX score group (low logistic clinical SYNTAX score, 2.9\%; high logistic clinical SYNTAX score, $15.7 \% ; p=.0016$, Figure $2 b)$. The logistic clinical SYNTAX score tended to improve risk classification for the 2-year all-cause mortality, compared to the anatomical CABG SYNTAX score (NRI, 0.221 [-0.068-0.511], $p=.134$ ) But there was no difference between the logistic clinical SYNTAX score and clinical SYNTAX score (NRI, 0.000 [-0.217-0. 217], $p=1.000$ ). The ROC curves of the anatomical CABG SYNTAX score, clinical SYNTAX score and logistic clinical SYNTAX score for the 2-year all-cause mortality are shown in Figure 3a, with significantly different AUC values of 0.582 (0.473-0.691), 0.721 (0.594-0.848), and 0.806 (0.714-0.899), respectively. This superior predictive ability of the logistic clinical SYNTAX score, compared to the anatomical CABG SYNTAX score and clinical SYNTAX score, was also demonstrated by the fact that IDI was $0.121(0.052-0.190, p<.001)$ and $0.052(0.010-0.094, p=.017)$, respectively.

The updated logistic clinical SYNTAX score systematically underestimated 2-year all-cause mortality as demonstrated by the positive 

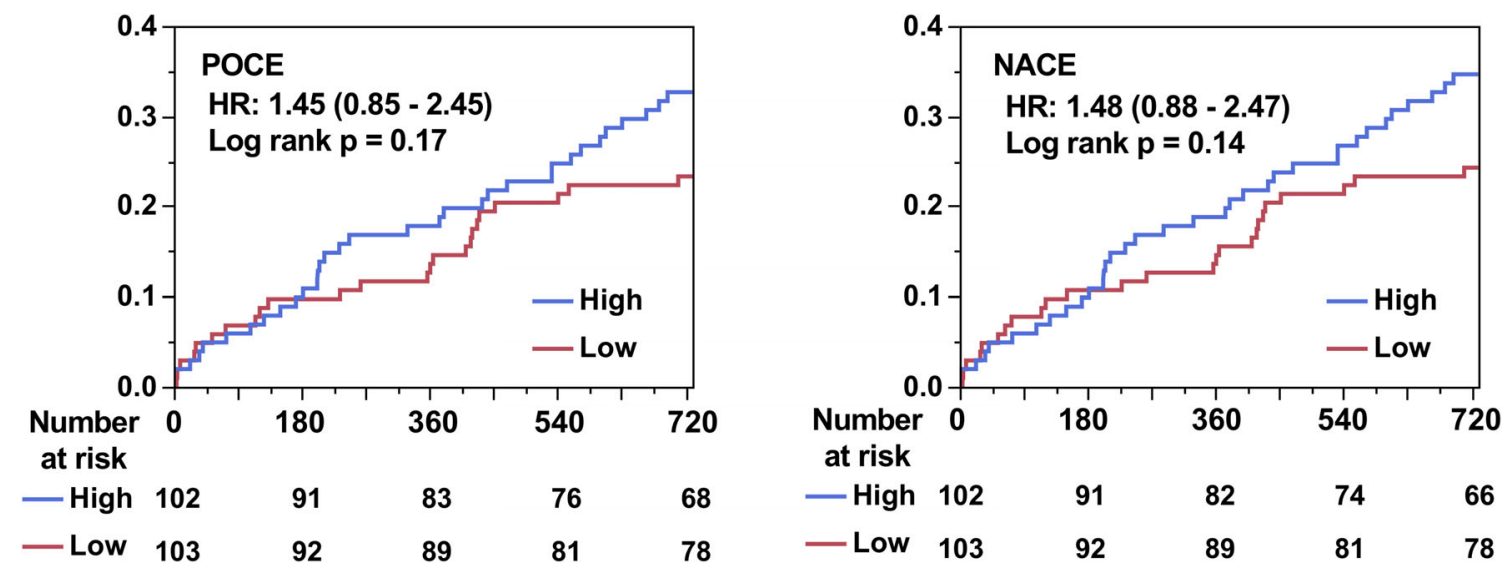

FIGURE 4 Cumulative incidence of patient-oriented composite endpoint (POCE) and net adverse clinical events (NACE) at 2 years [Color figure can be viewed at wileyonlinelibrary.com]

intercept (Figure 3b). Predicted probabilities of 2-year all-cause mortality in the two groups were close to the ideal line. The Brier score of the updated logistic clinical SYNTAX score for 2-year all-cause mortality was 0.076 .

Definite cardiovascular deaths were more frequently observed than noncardiovascular death $(n=11$ [5.37\%] vs. $n=7$ [3.37\%], respectively, Table 4). Of note, no patient died from definite cardiovascular cause in low logistic clinical SYNTAX score group (Table 4), and the predictive ability for the 2-year cardiovascular death of the logistic clinical SYNTAX score was high (AUC: 0.825 [0.717-0.934], Table 5).

Applying the logistic clinical SYNTAX score to the 2-year POCE and NACE, the rates of POCE and NACE in 2 years was not significantly different in low and high score groups (POCE: $23.3 \%$ vs. $32.7 \%$, $p=.17$; NACE: $24.2 \%$ vs. $34.6 \%, p=.14$, respectively, Figure 4). The AUC values for the 2-year POCE and NACE were 0.590 (0.496-0.685) and 0.592 (0.500-0.685), respectively. AUC values of components of these composite endpoints are shown in Table 5.

\section{4 | DISCUSSION}

The main finding of this study is that compared to the anatomical CABG SYNTAX score, the logistic clinical SYNTAX score is more effective in predicting 2 years all-cause mortality after $\mathrm{PCl}$ in patients with prior CABG. Furthermore, to the best of our knowledge, this analysis is the first to evaluate the predictive value of the logistic clinical SYNTAX score in patients with prior CABG.

Initially, we evaluated the performance of the anatomical CABG SYNTAX score in predicting 2-year all-cause mortality. An anatomical SYNTAX score $\leqq 22$ is generally accepted as a low score, ${ }^{22}$ and even in patients with prior $C A B G$, patients with a low anatomical CABG SYNTAX score $(\leqq 22)$ tended to be at low risk for death (Figure $2 b$ ). The anatomical CABG SYNTAX score in patients with prior CABG at the time of $\mathrm{PCl}$ reflects on one hand the anatomical complexity of coronary artery disease and on the other hand the extent and functionality of the surgical revascularization previously performed, but apparently cannot predict the 2 year vital prognosis of the planned percutaneous revascularization post CABG. Therefore, this score may not have a sufficient discriminative ability to predict 2 year mortality. At variance with the anatomical CABG SYNTAX score, the native anatomical SYNTAX score in patients with prior CABG reflects the overall atherosclerosic burden but does not reflect the extent and functionality of revascularization.

But, when patients were divided into two groups based on the median of the native anatomical SYNTAX score in the present study population (native anatomical SYNTAX score; $\leqq 34$ [low group, $n=105$ ], >34 [high group, $n=100$ ], Figure S1a), there was no significant difference in the rate of all-cause mortality at 2 years between patients in the low and high native anatomical SYNTAX score groups (low $=8.6 \%$ vs. high $=10.0 \%, p=.73$, Figure S1b).

Patients with equivalent anatomical SYNTAX scores sometimes have very different outcomes after revascularization, depending on the presence of comorbidities. ${ }^{23}$ To overcome this limitation, comorbidities derived from surgical scores such as the $\mathrm{ACEF}^{16,17}$ or EuroSCORE ${ }^{24}$ were incorporated into the anatomical SYNTAX score (Clinical SYNTAX score ${ }^{7}$ or Global risk classification, ${ }^{25}$ respectively). Following this, the logistic clinical SYNTAX score was developed, updated and validated by combining the anatomical SYNTAX score with clinical factors selected on the basis of logistic regression coefficients. ${ }^{8-10}$ Reflecting the logistic evolution of SYNTAX-derived scores, the predictive value for the 2-year all-cause mortality of the logistic clinical SYNTAX score was superior to the clinical SYNTAX score in this study (Figure 3a). Although the updated logistic clinical SYNTAX score systematically underestimated 2-year all-cause mortality in patients with prior CABG, predicted probabilities of 2-year all-cause mortality of the two groups were close to the identity line between the predicted and observed mortality (Figure $3 b$ ).

The discriminative ability of the logistic clinical SYNTAX score for 2-year all-cause mortality in 3271 patients without prior CABG has been already reported using the GLOBAL LEADERS database. ${ }^{10}$ Notably in this population, the prognostic value of the logistic clinical 
SYNTAX score was much higher in patients with prior CABG (AUC, 0.806; Figure 3a) than in patients without (0.71). ${ }^{10}$

This differential performance can be partially explained by considering the clinical characteristics and event rates in patients with and without prior CABG. The rate of all-cause mortality in the present study (Figure $2 \mathrm{~b}$ ) was $9.27 \%$, whereas in the 3,271 patients without prior CABG in the validation cohort it was $2.66 \% .{ }^{10}$ Patients with prior CABG also tended to be older, have lower $\mathrm{CrCl}$ and LVEF, and had more comorbidities, such as diabetes mellitus and peripheral vascular disease, compared to those without (Table 6). ${ }^{10}$ Furthermore, the rates of hypertension, hypercholesterolemia, previous $\mathrm{MI}$, previous $\mathrm{PCl}$, previous stroke, and COPD were also higher in patients with prior CABG, compared to those without (Table 6). Taken together, the difference between the predictive values of the logistic clinical SYNTAX score in patients with prior CABG compared to those without might be largely dependent on major differences in clinical characteristics.

The predictive values of the logistic clinical SYNTAX score for POCE and NACE were poor mainly due to the poor predictive value for revascularization, although those for any stroke and myocardial infarction were possibly helpful. ${ }^{26}$ The logistic clinical SYNTAX score was updated to predict all-cause mortality in 6304 patients enrolled in seven contemporary coronary stent trials (SIRTAX, ARTS-II, STRATEGY, MULTISTRATEGY, LEADERS, SYNTAX, RESOLUTE All-Comers), and was not accurate enough to predict outcomes other than mortality. However, of note, in the present study, all definite cardiovascular deaths occurred in the high logistic clinical SYNTAX score group (Table 4).

Pharmacological therapy and lifestyle changes for risk factor modification has been strongly recommended for secondary prevention

TAB LE 6 Patient characteristics in patients with and without prior $\mathrm{CABG}$

\begin{tabular}{|c|c|c|c|}
\hline \multirow[b]{2}{*}{ Baseline characteristics } & Present study & \multirow[b]{2}{*}{$\begin{array}{l}\text { Patients without } \\
\text { prior CABG } \\
n=3,271\end{array}$} & \multirow[b]{2}{*}{$p$ value } \\
\hline & $\begin{array}{l}\text { Patients with } \\
\text { prior CABG } \\
n=205\end{array}$ & & \\
\hline Age (years) & $68.8 \pm 8.73$ & $64.3 \pm 10.5$ & $<.001$ \\
\hline Male & $83.4 \%$ & $76.4 \%$ & .021 \\
\hline Body mass index $\left(\mathrm{kg} / \mathrm{m}^{2}\right)$ & $28.2 \pm 4.73$ & $28.1 \pm 4.46$ & .923 \\
\hline $\mathrm{CrCl}(\mathrm{ml} / \mathrm{min})$ & $81.0 \pm 28.8$ & $92.8 \pm 32.7$ & $<.001$ \\
\hline LVEF (\%) & $52.3 \pm 12.6$ & $54.8 \pm 10.7$ & .007 \\
\hline Hypertension & $82.9 \%$ & $69.7 \%$ & $<.001$ \\
\hline Hypercholesterolemia & $87.2 \%$ & $66.9 \%$ & $<.001$ \\
\hline Diabetes mellitus & $36.6 \%$ & $22.9 \%$ & $<.001$ \\
\hline Previous MI & $46.1 \%$ & $20.9 \%$ & $<.001$ \\
\hline Previous $\mathrm{PCl}$ & $52.7 \%$ & $28.6 \%$ & $<.001$ \\
\hline Previous stroke & $4.88 \%$ & $2.33 \%$ & .034 \\
\hline Established PVD & $20.0 \%$ & $6.14 \%$ & $<.001$ \\
\hline COPD & $9.76 \%$ & $5.61 \%$ & .020 \\
\hline
\end{tabular}

Note: Continuous variables were expressed as mean $\pm S D$, and categorical variables were reported as percentages. $\mathrm{CrCl}$, creatinine clearance; LVEF, left ventricular ejection fraction; $\mathrm{Ml}$, myocardial infarction; $\mathrm{PCl}$, percutaneous coronary intervention; PVD, peripheral vascular disease; COPD, chronic obstructive pulmonary disease. (la). ${ }^{22}$ In the SYNTAX trial started in 2005 , optimal medical therapy (OMT), defined as the combination of at least 1 antiplatelet, angiotensin-converting-enzyme (ACE)-inhibitor/ angiotensin II receptor blocker (ARB), beta-blocker, and statin was given in only $50.2 \%$ of patients at the time of discharge after $\mathrm{PCl}^{27}$ The GLOBAL LEADERS trial was started in 2013. Regardless of the strong OMT recommendation, OMT was prescribed in $61.6 \%$ at discharge and even in the high logistic clinical SYNTAX score patients, the rate of OMT was almost the same and only 60\% (Table 3). The logistic clinical SYNTAX score can predict the individual mortality rate after $\mathrm{PCl}$, and should be a strong incentive to an aggressive adjunctive pharmacological treatment and a closer monitoring of these patients at high risk.

In the future, further iterations of the logistic clinical SYNTAX score may be needed due to improvement of mortality after $\mathrm{PCI}$. However, at present, the logistic clinical SYNTAX score has a high predictive ability for 2-year all-cause mortality after $\mathrm{PCl}$ in patients with prior $C A B G$.

\section{1 | Limitation}

The present study is based on a non-prespecified post hoc analysis. In view of the post hoc nature of the analysis, the results have to be interpreted strictly as hypothesis-generating. The sample size ( $n=205)$ was small as a subanalysis of the large GLOBAL LEADERS trial $(n=15,991)$. The number of deaths was 19 in the present study, and external validation of a prognostic model generally requires a minimum of 100 events. ${ }^{28}$ Therefore, the sample size might be insufficient to demonstrate the efficacy of the anatomical CABG SYNTAX score. From the 275 patients with prior CABG included in the first 4,000 consecutive patients with corelab analysis of the SYNTAX score (prespecified analysis), We excluded patients without anatomical CABG SYNTAX score and patients who had at least one missing variable for the calculation of the logistic clinical SYNTAX score. Therefore, selection bias might exist. The results need to be confirmed in dedicated large-scale trials. In terms of extent of revascularization, the higher prognostic value of the post- $\mathrm{PCl}$ (residual) CABG SYNTAX score in patients with prior CABG, compared to the CABG SYNTAX score, has been previously reported. ${ }^{6}$ In the GLOBAL LEADERS trial, only diagnostic angiograms for index $\mathrm{PCl}$ were collected and post procedural angiograms were not available, therefore, the predictivity of the residual CABG SYNTAX score could not be evaluated. In addition, detailed information about prior CABG surgery, such as the number of bypass grafts performed and the completeness of revascularization, was missing in this trial which enrolled patients exclusively for $\mathrm{PCl}$.

\section{5 | CONCLUSION}

The logistic clinical SYNTAX score was superior to the anatomical CABG SYNTAX score for predicting 2-year all-cause mortality after $\mathrm{PCl}$. 


\section{CONFLICT OF INTEREST}

Dr. Hara is supported by a grant for studying overseas from Japanese Circulation Society and a grant from Fukuda Foundation for Medical Technology. Dr. Modolo reports research grant from the Sao Paulo Research Foundation (FAPESP grant number 2017/22013-8) and Biosensors. Dr. Geisler reports personal fees from Astra Zeneca, personal fees from Boehringer Ingelheim, personal fees from Pfizer, grants and personal fees from Bayer Healthcare, grants and personal fees from Bristol Myers Squibb, grants and personal fees from Daiichi Sankyo, grants and personal fees from Eli Lilly, personal fees from Ferrer, outside the submitted work. Dr. Jüni serves as unpaid member of the steering group of trials funded by Astra Zeneca, Biotronik, Biosensors, St. Jude Medical and The Medicines Company, has received research grants to the institution from Astra Zeneca, Biotronik, Biosensors International, Eli Lilly and The Medicines Company, and honoraria to the institution for participation in advisory boards from Amgen, but has not received personal payments by any pharmaceutical company or device manufacturer. Dr. Hamm reports personal fees from AstraZeneca. Dr. Steg reports grants and personal fees from Bayer/Janssen, grants and personal fees from Merck, grants and personal fees from Sanofi, grants and personal fees from Amarin, personal fees from Amgen, personal fees from Bristol Myers Squibb, personal fees from Boehringer-Ingelheim, personal fees from Pfizer, personal fees from Novartis, personal fees from Regeneron, personal fees from Lilly, personal fees from AstraZeneca, grants and personal fees from Servier, personal fees from Idorsia, outside the submitted work. Dr. Valgimigli reports personal fees from Astra Zeneca, grants and personal fees from Terumo, personal fees from Alvimedica/CID, personal fees from Abbott Vascular, personal fees from Daiichi Sankyo, personal fees from Opsens, personal fees from Bayer, personal fees from CoreFLOW, personal fees from IDORSIA PHARMACEUTICALS LTD, personal fees from Universität Basel, Dept. Klinische Forschung, personal fees from Vifor, personal fees from Bristol Myers Squib SA, personal fees from iVascular, outside the submitted work. Dr. Vranckx reports personal fees from AstraZeneca and the Medicines Company during the conduct of the study and personal fees from Bayer Health Care, Terumo, and Daiichi-Sankyo outside the submitted work. Dr. Windecker reports research and educational grants to the institution from Abbott, Amgen, Biotronik, Bayer, BMS, Boston Scientific, CSL Behring, Edwards Lifesciences, Medtronic, Polares and Sinomed. Dr. Serruys reports personal fees from Biosensors, Micel Technologies, Sinomedical Sciences Technology, Philips/Volcano, Xeltis, and HeartFlow, outside the submitted work. All other authors have no conflict of interest to declare.

\section{ORCID}

Mariusz Tomaniak (DD https://orcid.org/0000-0001-8289-1393

Masafumi Ono (D) https://orcid.org/0000-0002-3919-5648

Hideyuki Kawashima (D) https://orcid.org/0000-0002-2847-1248

Marco Valgimigli (D) https://orcid.org/0000-0002-4353-7110

\section{REFERENCES}

1. Sianos G, Morel MA, Kappetein AP, et al. The SYNTAX score: an angiographic tool grading the complexity of coronary artery disease. Eurolntervention. 2005;1(2):219-227.
2. Serruys PW, Onuma $Y$, Garg $S$, et al. Assessment of the SYNTAX score in the syntax study. Eurolntervention. 2009;5(1):50-56.

3. Serruys PW, Morice MC, Kappetein AP, et al. Percutaneous coronary intervention versus coronary-artery bypass grafting for severe coronary artery disease. N Engl J Med. 2009;360(10):961-972.

4. Serruys PW, Farooq V, Vranckx P, et al. A global risk approach to identify patients with left main or 3-vessel disease who could safely and efficaciously be treated with percutaneous coronary intervention: the SYNTAX Trial at 3 years. JACC Cardiovasc Interv. 2012;5(6): 606-617.

5. Farooq V, Girasis C, Magro M, et al. The CABG SYNTAX Score - an angiographic tool to grade the complexity of coronary disease following coronary artery bypass graft surgery: from the SYNTAX Left Main Angiographic (SYNTAX-LE MANS) substudy. Eurolntervention. 2013; 8(11):1277-1285

6. Miyagi T, Asaumi $\mathrm{Y}$, Nishimura K, et al. Validation of the coronary artery bypass graft SYNTAX score (synergy between percutaneous coronary intervention with Taxus) as a prognostic marker for patients with previous coronary artery bypass graft surgery after percutaneous coronary intervention. Circ Cardiovasc Interv. 2016;9(9):e003459.

7. Garg S, Sarno G, Garcia-Garcia HM, et al. A new tool for the risk stratification of patients with complex coronary artery disease: the clinical SYNTAX score. Circ Cardiovasc Interv. 2010;3(4):317-326.

8. Farooq V, Vergouwe $\mathrm{Y}$, Raber L, et al. Combined anatomical and clinical factors for the long-term risk stratification of patients undergoing percutaneous coronary intervention: the Logistic Clinical SYNTAX score. Eur Heart J. 2012;33(24):3098-3104.

9. Iqbal J, Vergouwe $\mathrm{Y}$, Bourantas CV, et al. Predicting 3-year mortality after percutaneous coronary intervention: updated logistic clinical SYNTAX score based on patient-level data from 7 contemporary stent trials. JACC Cardiovasc Interv. 2014;7(5):464-470.

10. Chichareon $P$, Onuma $Y$, van Klaveren $D$, et al. Validation of the updated logistic clinical SYNTAX score for all-cause mortality in the GLOBAL LEADERS trial. Eurolntervention. 2019;15(6):e539-e546.

11. Capodanno D, Giacoppo D, Dipasqua F, et al. Usefulness of the logistic clinical SYNTAX score for predicting 1-year mortality in patients undergoing percutaneous coronary intervention of the left main coronary artery. Catheter Cardiovasc Interv. 2013;82(4):E446-E452.

12. Farooq V, Vergouwe $\mathrm{Y}$, Genereux P, et al. Prediction of 1-year mortality in patients with acute coronary syndromes undergoing percutaneous coronary intervention: validation of the logistic clinical SYNTAX (synergy between percutaneous coronary interventions with Taxus and cardiac surgery) score. JACC Cardiovasc Interv. 2013;6(7): 737-745.

13. Vranckx $P$, Valgimigli $M$, Juni $P$, et al. Ticagrelor plus aspirin for 1 month, followed by ticagrelor monotherapy for 23 months vs aspirin plus clopidogrel or ticagrelor for 12 months, followed by aspirin monotherapy for 12 months after implantation of a drug-eluting stent: a multicentre, open-label, randomised superiority trial. Lancet. 2018;392(10151):940-949.

14. Vranckx $P$, Valgimigli $M$, Windecker $S$, et al. Long-term ticagrelor monotherapy versus standard dual antiplatelet therapy followed by aspirin monotherapy in patients undergoing biolimus-eluting stent implantation: rationale and design of the GLOBAL LEADERS trial. Eurolntervention. 2016;12(10):1239-1245.

15. Leaman DM, Brower RW, Meester GT, Serruys P, van den Brand M. Coronary artery atherosclerosis: severity of the disease, severity of angina pectoris and compromised left ventricular function. Circulation. 1981;63(2):285-299.

16. Ranucci M, Castelvecchio S, Menicanti L, Frigiola A, Pelissero G. Risk of assessing mortality risk in elective cardiac operations: age, creatinine, ejection fraction, and the law of parsimony. Circulation. 2009; 119(24):3053-3061.

17. Chichareon $\mathrm{P}$, Modolo R, van Klaveren $\mathrm{D}$, et al. Predictive ability of ACEF and ACEF II score in patients undergoing percutaneous 
coronary intervention in the GLOBAL LEADERS study. Int J Cardiol. 2019;286:43-50.

18. Serruys PW, Chichareon P, Modolo R, et al. The SYNTAX score on its way out or ... towards artificial intelligence: part I. Eurolntervention. 2019. https://doi.org/10.4244/EIJ-D-19-00543A. [Epub ahead of print].

19. Serruys PW, Chichareon P, Modolo R, et al. The SYNTAX score on its way out or ... towards artificial intelligence: part II. Eurolntervention. 2019. https://doi.org/10.4244/EIJ-D-19-00543B. [Epub ahead of print].

20. Garcia-Garcia HM, McFadden EP, Farb A, et al. Standardized end point definitions for coronary intervention trials: the Academic Research Consortium-2 consensus document. Eur Heart J. 2018;39 (23):2192-2207.

21. Steyerberg EW, Vergouwe Y. Towards better clinical prediction models: seven steps for development and an $A B C D$ for validation. Eur Heart J. 2014;35(29):1925-1931.

22. Neumann FJ, Sousa-Uva M, Ahlsson A, et al. 2018 ESC/EACTS Guidelines on myocardial revascularization. Eur Heart J. 2019;40(2): 87-165.

23. Hara $\mathrm{H}$, Aoki J, Tanabe $\mathrm{K}$, et al. Impact of the clinical syntax score on 5-year clinical outcomes after sirolimus-eluting stents implantation. Cardiovasc Interv Ther. 2013;28(3):258-266.

24. Nashef SA, Roques F, Michel P, Gauducheau E, Lemeshow S, Salamon R. European system for cardiac operative risk evaluation (EuroSCORE). Eur J Cardiothorac Surg. 1999;16(1):9-13.

25. Capodanno D, Miano M, Cincotta G, et al. EuroSCORE refines the predictive ability of SYNTAX score in patients undergoing left main percutaneous coronary intervention. Am Heart J. 2010;159(1): 103-109.
26. Alba AC, Agoritsas T, Walsh M, et al. Discrimination and calibration of clinical prediction models: users' guides to the medical literature. JAMA. 2017;318(14):1377-1384.

27. Iqbal J, Zhang YJ, Holmes DR, et al. Optimal medical therapy improves clinical outcomes in patients undergoing revascularization with percutaneous coronary intervention or coronary artery bypass grafting: insights from the Synergy Between Percutaneous Coronary Intervention with TAXUS and cardiac surgery (SYNTAX) trial at the 5-year follow-up. Circulation. 2015;131(14):1269-1277.

28. Collins GS, Ogundimu EO, Altman DG. Sample size considerations for the external validation of a multivariable prognostic model: a resampling study. Stat Med. 2016;35(2):214-226.

\section{SUPPORTING INFORMATION}

Additional supporting information may be found online in the Supporting Information section at the end of this article.

How to cite this article: Hara H, Kogame N, Takahashi K, et al. Usefulness of the updated logistic clinical SYNTAX score after percutaneous coronary intervention in patients with prior coronary artery bypass graft surgery: Insights from the GLOBAL LEADERS trial. Catheter Cardiovasc Interv. 2020;

1-11. https://doi.org/10.1002/ccd.28898 